\title{
Bivariate genome-wide association study identifies novel pleiotropic loci for lipids and inflammation
}

Symen Ligthart ${ }^{1}$, Ahmad Vaez ${ }^{2}$, Yi-Hsiang Hsu ${ }^{3,4}$, Inflammation Working Group of the CHARGE Consortium, PMIWG-XCP, LifeLines Cohort Study, Ronald Stolk², André G. Uitterlinden ${ }^{6}$, Albert Hofman', Behrooz Z. Alizadeh², Oscar H. Franco ${ }^{1}$ and Abbas Dehghan ${ }^{1 *}$

\begin{abstract}
Background: Genome-wide association studies (GWAS) have identified multiple genetic loci for C-reactive protein (CRP) and lipids, of which some overlap. We aimed to identify genetic pleiotropy among CRP and lipids in order to better understand the shared biology of chronic inflammation and lipid metabolism.

Results: In a bivariate GWAS, we combined summary statistics of published GWAS on CRP $(n=66,185)$ and lipids, including LDL-cholesterol, HDL-cholesterol, triglycerides, and total cholesterol $(n=100,184)$, using an empirical weighted linear-combined test statistic. We sought replication for novel CRP associations in an independent sample of 17,743 genotyped individuals, and performed in silico replication of novel lipid variants in 93,982 individuals. Fifty potentially pleiotropic SNPs were identified among CRP and lipids: 21 for LDL-cholesterol and CRP, 20 for HDL-cholesterol and CRP, 21 for triglycerides, and CRP and 20 for total cholesterol and CRP. We identified and significantly replicated three novel SNPs for CRP in or near CTSB/FDFT1 (rs10435719, Preplication: $2.6 \times 10^{-5}$ ), STAG1/PCCB (rs7621025, Preplication: $1.4 \times 10^{-3}$ ) and FTO (rs1558902, Preplication: $\left.2.7 \times 10^{-5}\right)$. Seven pleiotropic lipid loci were replicated in the independent set of MetaboChip samples of the Global Lipids Genetics Consortium. Annotating the effect of replicated CRP SNPs to the expression of nearby genes, we observed an effect of rs10435719 on gene expression of FDFT1, and an effect of rs7621025 on PCCB.

Conclusions: Our large scale combined GWAS analysis identified numerous pleiotropic loci for CRP and lipids providing further insight in the genetic interrelation between lipids and inflammation. In addition, we provide evidence for FDFTI, PCCB and FTO to be associated with CRP levels.
\end{abstract}

Keywords: C-reactive protein, Inflammation, Lipids, Genome-wide association study, Genetic pleiotropy

\section{Background}

Genome-wide association studies (GWAS) have identified hundreds of genetic loci for cardiovascular disease and it's risk factors, including chronic inflammation and lipids [1-3]. Some of the identified genetic variants are associated with more than one phenotype, termed genetic pleiotropy [4]. Examples are APOC1(rs4420638) and HNF1A (rs1183910), which are associated both with lipids and C-reactive protein (CRP) $[2,3]$. As randomized

\footnotetext{
* Correspondence: a.dehghan@erasmusmc.nl

${ }^{1}$ Department of Epidemiology, Erasmus University Medical Center, PO Box 20403000CA Rotterdam, The Netherlands

Full list of author information is available at the end of the article
}

clinical trials have shown a coextending effect of statin treatment on the lowering of LDL-cholesterol and CRP, we do expect inflammation and lipids to share certain biological pathways $[5,6]$. Moreover, there is accumulating evidence that the pleiotropic effects are partially independent, although the biological mechanisms are not fully understood [7]. The identification of further pleiotropic genes could provide insight into the biological mechanisms that link chronic inflammation to lipids.

Therefore, we aimed to identify further shared genes for lipids and CRP. In order to enhance the statistical power of genetic studies to find pleiotropic genes for the correlated phenotypes of interest, we applied a method 
that combines GWAS meta-analysis summary statistics allowing for mixed directions of effect, a common observed phenomenon in genetic pleitropy [8]. In a second step we sought to replicate novel associations with lipids and CRP in an independent sample of 93,982 genotyped individuals for lipids and 17,743 genotyped individuals for CRP. We identified multiple overlapping genetic variants between CRP and lipids and confirmed novel genes implicated in the biology of chronic inflammation.

\section{Results}

\section{Bivariate genome-wide association analysis}

We performed bivariate GWAS meta-analyses by combining summary statistics ( $Z$ test statistics) from the univariate GWAS of CRP pairing with the summary statistics of each GWAS of the lipid phenotypes, using an empirical-weighted linear-combined test statistics (eLC) [8]. This method allows mixed genetic effects in the univariate phenotype GWAS, a phenomenon commonly observed in genetic studies.

\section{CRP and LDL-cholesterol}

Manhattan plots for the bivariate GWAS are depicted in Fig. 1. Table 1 indicates the results from the bivariate analysis combining CRP and LDL-cholesterol genetic association data. The bivariate analysis resulted in 21 potentially pleiotropic loci. We identified fourteen loci associated with CRP levels which had no genome-wide significant SNP in the original GWAS of CRP. These potential novel associations were located in or near CELSR2, IRF2BP2, ABCG8, GCNT4, HLA-DQB1, FRK,
TRIB1, FADS2, ST3GAL4, BRAP, C12orf51, CARM1/ $L D L R, N C A N$ and RASIP1. The potential novel associations for LDL-cholesterol were located in or near GCKR, IL1F10, RORA, RASIP1 and in HNF4A. The SNPs identified in the bivariate GWAS near $H L A-D Q B 1, F R K$, $B R A P, c 12$ orf51 and CARM1/LDLR were not genomewide significant in the original univariate GWAS on LDL-cholesterol, however other SNPs in their vicinity were significant in the original GWAS on LDLcholesterol and the loci have thus been reported previously. The variants in and near PPP1R3B, HNF1A and $A P O C 1$ were already genome-wide significant in both GWAS of CRP and LDL-cholesterol.

\section{CRP and HDL-cholesterol}

We identified 20 potential pleiotropic SNPs (Table 2). The variants near CELSR2, STAG1, HLA-DRA, JMJD1C, FADS1, LIPC, CETP, LYPLA3, LIPG and MC4R were not genome-wide significant in the original CRP metaGWAS analysis. Seven SNPs were potentially novel for both CRP and HDL-cholesterol: the SNP rs12742376 located in C1orf172 on chromosome $1\left(P_{\text {bivariate }}=1.4 \times 10^{-8}\right)$, rs7621025 in STAG1 on chromosome $3\left(P_{\text {bivariate }}=1.2 \times 10^{-9}\right)$, rs937 8212 near HLA-DRA $\left(P_{\text {bivariate }}=6.7 \times 10^{-10}\right)$, rs10761731 in JMJD1C $\left(P_{\text {bivariate }}=2.2 \times 10^{-8}\right)$, rs1936797 in RSPO3 on chromosome $6\left(P_{\text {bivariate }}=6.7 \times 10^{-9}\right)$, rs4871137 near SNTB1 $\left(P_{\text {bivariate }}=3.3 \times 10^{-8}\right)$ on chromosome 8 and the FTO SNP rs1558902 $\left(P_{\text {bivariate }}=5.0 \times 10^{-9}\right)$ on chromosome 16 . The variants near CELSR2 and PLTP were not significant in the original GWAS on HDL-cholesterol, but these loci were identified in the original GWAS. The variants in or

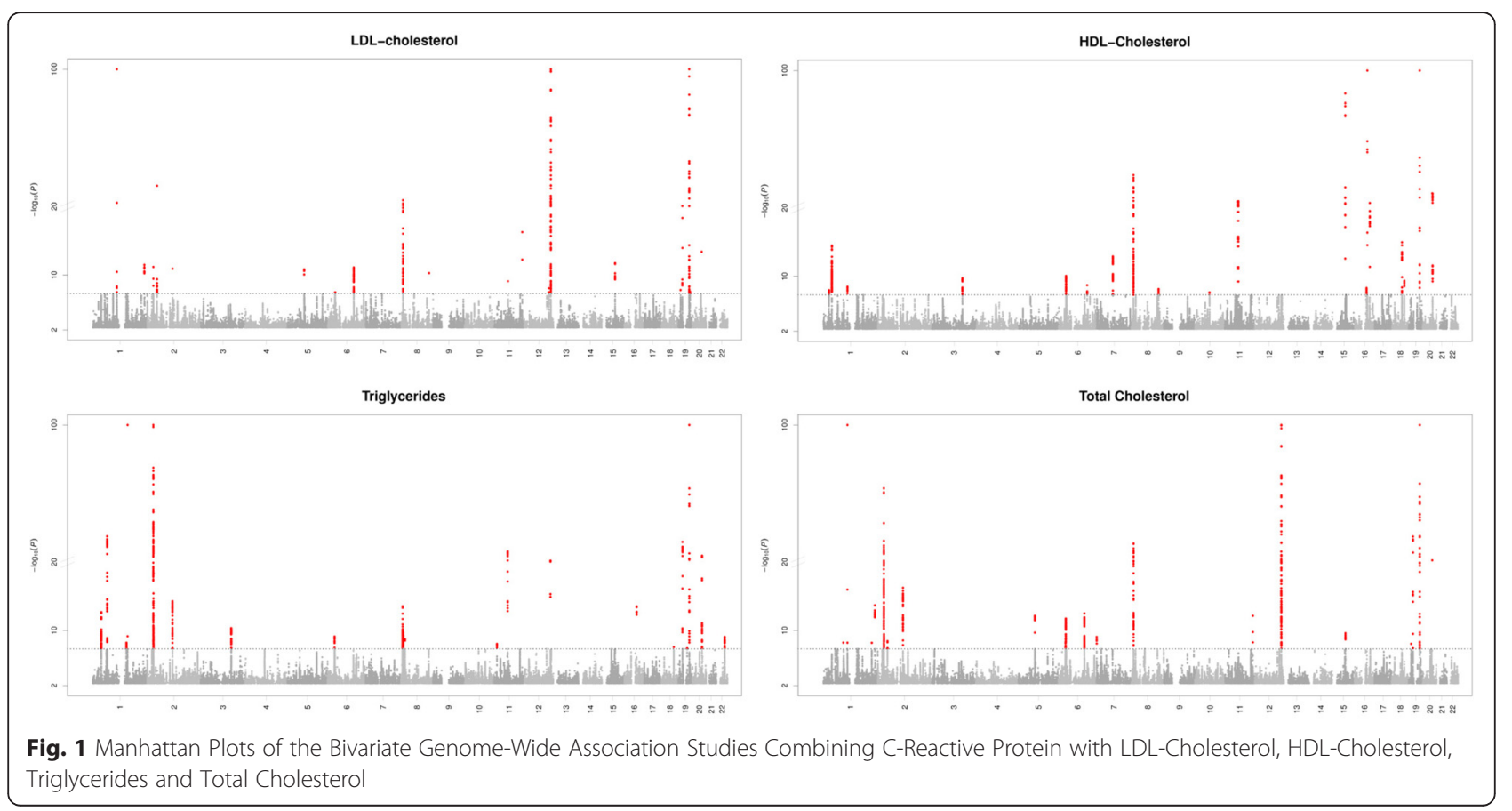


Table 1 Results of Bivariate GWAS for C-Reactive Protein and LDL-Cholesterol Levels

\begin{tabular}{|c|c|c|c|c|c|c|c|c|c|}
\hline \multirow[t]{2}{*}{ SNP } & \multirow[t]{2}{*}{ Chromosome } & \multirow[t]{2}{*}{ Position } & \multirow{2}{*}{$\begin{array}{l}\text { Effect } \\
\text { Allele }\end{array}$} & \multicolumn{2}{|c|}{ C-reactive protein } & \multicolumn{2}{|c|}{ LDL-cholesterol } & \multirow{2}{*}{$\begin{array}{l}\text { Pleiotropy } \\
\text { significance }\end{array}$} & \multirow[t]{2}{*}{ Gene } \\
\hline & & & & Beta & P-value & Beta & P-value & & \\
\hline rs646776 & 1 & 109620053 & $\mathrm{~T}$ & -0.018 & 0.02 & 0.171 & $4.5 \times 10^{-169}$ & $4.3 \times 10^{-170}$ & CELSR2 \\
\hline rs661955 & 1 & 232909479 & C & -0.021 & $1.7 \times 10^{-3}$ & 0.034 & $1.2 \times 10^{-10}$ & $3.2 \times 10^{-12}$ & IRF2BP2 \\
\hline rs3817588 & 2 & 27584716 & $T$ & 0.053 & $1.8 \times 10^{-10}$ & 0.024 & $4.2 \times 10^{-4}$ & $6.4 \times 10^{-12}$ & GCKR \\
\hline rs11887534 & 2 & 43919751 & C & -0.049 & $2.5 \times 10^{-4}$ & -0.134 & $1.1 \times 10^{-31}$ & $9.0 \times 10^{-33}$ & $A B C G 8$ \\
\hline rs12711751 & 2 & 113554236 & $\mathrm{~T}$ & -0.044 & $1.6 \times 10^{-10}$ & 0.014 & $4.8 \times 10^{-3}$ & $1.2 \times 10^{-11}$ & IL1F10 \\
\hline rs4703642 & 5 & 74297918 & $A$ & 0.018 & $3.0 \times 10^{-3}$ & -0.031 & $3.1 \times 10^{-10}$ & $1.5 \times 10^{-11}$ & GCNT4 \\
\hline rs9275292 & 6 & 32771267 & $A$ & 0.022 & $3.6 \times 10^{-4}$ & 0.023 & $1.1 \times 10^{-5}$ & $3.3 \times 10^{-8}$ & HLA-DQB1 \\
\hline rs3822857 & 6 & 116420624 & C & -0.032 & $2.7 \times 10^{-6}$ & -0.030 & $2.3 \times 10^{-7}$ & $7.6 \times 10^{-12}$ & FRK \\
\hline rs9987289 & 8 & 9220768 & $A$ & -0.079 & $2.1 \times 10^{-12}$ & -0.071 & $2.0 \times 10^{-14}$ & $2.3 \times 10^{-24}$ & PPP1R3B \\
\hline rs8180991 & 8 & 126569532 & $C$ & -0.026 & $9.0 \times 10^{-4}$ & -0.041 & $8.0 \times 10^{-10}$ & $5.1 \times 10^{-11}$ & TRIB1 \\
\hline rs174574 & 11 & 61356918 & A & -0.027 & $1.7 \times 10^{-3}$ & -0.050 & $1.1 \times 10^{-8}$ & $7.8 \times 10^{-10}$ & FADS2 \\
\hline rs11220463 & 11 & 125753421 & A & 0.032 & $2.8 \times 10^{-3}$ & -0.070 & $1.3 \times 10^{-15}$ & $5.8 \times 10^{-17}$ & ST3GAL4 \\
\hline rs10744775 & 12 & 110580598 & $\mathrm{~T}$ & 0.021 & $4.0 \times 10^{-3}$ & -0.030 & $5.3 \times 10^{-7}$ & $3.1 \times 10^{-8}$ & BRAP \\
\hline rs2285810 & 12 & 111183923 & $\mathrm{~T}$ & 0.019 & $6.8 \times 10^{-3}$ & -0.030 & $8.3 \times 10^{-8}$ & $8.3 \times 10^{-9}$ & C12orf51 \\
\hline rs1183910 & 12 & 119905190 & A & -0.151 & $4.6 \times 10^{-113}$ & 0.042 & $5.8 \times 10^{-15}$ & $5.6 \times 10^{-128}$ & HNFIA \\
\hline rs340005 & 15 & 58665322 & A & 0.044 & $3.2 \times 10^{-11}$ & -0.015 & $3.4 \times 10^{-3}$ & $1.7 \times 10^{-12}$ & RORA \\
\hline rs1529711 & 19 & 10884434 & $\mathrm{~T}$ & 0.030 & $8.4 \times 10^{-4}$ & 0.037 & $1.5 \times 10^{-6}$ & $1.5 \times 10^{-8}$ & CARM1/LDLR \\
\hline rs2228603 & 19 & 19190924 & $T$ & 0.036 & $2.9 \times 10^{-3}$ & -0.089 & $1.4 \times 10^{-19}$ & $6.5 \times 10^{-21}$ & NCAN \\
\hline rs4420638 & 19 & 50114786 & A & 0.240 & $1.0 \times 10^{-129}$ & -0.215 & $8.7 \times 10^{-147}$ & $1.2 \times 10^{-283}$ & APOC1 \\
\hline rs2287921 & 19 & 53920084 & $T$ & -0.019 & $3.6 \times 10^{-3}$ & -0.026 & $3.4 \times 10^{-7}$ & $2.8 \times 10^{-8}$ & RASIP1 \\
\hline rs1800961 & 20 & 42475778 & $\mathrm{~T}$ & -0.120 & $2.4 \times 10^{-11}$ & -0.070 & $2.4 \times 10^{-5}$ & $3.8 \times 10^{-14}$ & HNF4A \\
\hline
\end{tabular}

Abbreviations: SNP single nucleotide polymorphism

For both CRP and the lipid phenotype, the effect estimates are according to the original GWAS

Chromosome and position are in NCBI genome build 36

Beta coefficient for CRP represents 1-unit change in the natural log-transformed CRP $(\mathrm{mg} / \mathrm{L})$ per copy increment in the coded allele

Beta coefficient for LDL-cholesterol represents 1-unit change in the standardized LDL-cholesterol levels per copy increment in the coded allele

near PABPC4, BAZ1B, PPP1R3B, APOC1 and HNF4A were already genome-wide significant in both the CRP and HDL-cholesterol univariate GWAS.

\section{CRP and Triglycerides}

Table 3 lists the 21 potentially pleiotropic SNPs that were identified combining the GWAS results of triglycerides and CRP. For triglycerides, we identified eleven potential novel associations compared to the original GWAS located in or near PABPC4, LEPR, ADAR, CRP, IL1F10, PPP1R3B, CTSB/FDFT1, ARNTL, CABP1, $M C 4 R$ and HPN. The variant near PLA2G6 was not genome-wide significant in the original GWAS, but this locus was identified in the original GWAS. The variants in and near ADAR, MSL2L1, HLA-C, CTSB/FDFT1, LPL, ARNTL, FADS1, CETP, MC4R, SF4, HPN, ZNF335/ PLTP and PLA2G6 were potential novel associations with CRP level. Five loci were not genome-wide significant in either the original GWAS on CRP or triglycerides: the SNP rs1127311 within ADAR on chromosome 1 $\left(P_{\text {bivariate }}=6.4 \times 10^{-9}\right)$, rs10435719 located $77 \mathrm{~Kb}$ upstream of CTSB on chromosome $8 \quad\left(P_{\text {bivariate }}=2.0 \times 10^{-10}\right)$, rs10832027 located in the second intron of ARNTL on chromosome $11 \quad\left(P_{\text {bivariate }}=9.4 \times 10^{-9}\right)$, rs571312 on chromosome 18 near $\operatorname{MC} 4 R\left(P_{\text {bivariate }}=2.8 \times 10^{-8}\right)$, and the chromosome 19 rs1688043 in the fifth intron of HPN $\left(P_{\text {bivariate }}=4.1 \times 10^{-8}\right)$. In both the original GWAS of CRP and triglycerides, GCKR and APOC1 were already genome-wide significant.

\section{CRP and total cholesterol}

Twenty potentially pleiotropic SNPs were identified combining CRP and total cholesterol (Table 4). The SNPs in or near ZNF644, SLC44A4, C7orf50 and RORA were potentially novel for total cholesterol. The variants near HLX, ABCG5, IL1F10, C7orf60 and CARM1 were not genome-wide significant in the GWAS on total cholesterol, but the loci were identified in this original GWAS. For CRP, ZNF664, CELSR2, HLX, IRF2BP2, ABCG5, GCNT4, SLC44A4, HLA-DQB1, FRK, ST3GAL4, $C A R M 1$ and NCAN were potentially novel compared to the univariate GWAS. The SNPs near ZNF644 and 
Table 2 Results of Bivariate GWAS Analyses for C-Reactive Protein and HDL-Cholesterol Levels

\begin{tabular}{|c|c|c|c|c|c|c|c|c|c|}
\hline \multirow[t]{2}{*}{ SNP } & \multirow[t]{2}{*}{ Chromosome } & \multirow[t]{2}{*}{ Position } & \multirow{2}{*}{$\begin{array}{l}\text { Effect } \\
\text { Allele }\end{array}$} & \multicolumn{2}{|c|}{ C-reactive protein } & \multicolumn{2}{|c|}{ HDL-cholesterol } & \multirow{2}{*}{$\begin{array}{l}\text { Pleiotropy } \\
\text { significance }\end{array}$} & \multirow[t]{2}{*}{ Gene } \\
\hline & & & & Beta & P-value & Beta & P-value & & \\
\hline rs12742376 & 1 & 27157782 & $T$ & -0.027 & $1.7 \times 10^{-2}$ & -0.046 & $2.8 \times 10^{-7}$ & $1.4 \times 10^{-8}$ & Clorf172 \\
\hline rs4660293 & 1 & 39800767 & A & -0.044 & $1.2 \times 10^{-9}$ & 0.034 & $4.0 \times 10^{-10}$ & $3.1 \times 10^{-15}$ & PABPC4 \\
\hline rs646776 & 1 & 109620053 & $\mathrm{~T}$ & -0.018 & $1.8 \times 10^{-2}$ & -0.033 & $6.4 \times 10^{-8}$ & $3.2 \times 10^{-9}$ & CELSR2 \\
\hline rs7621025 & 3 & 137754936 & $\mathrm{~T}$ & 0.028 & $1.7 \times 10^{-4}$ & 0.026 & $4.1 \times 10^{-6}$ & $1.2 \times 10^{-9}$ & STAGI \\
\hline rs9378212 & 6 & 32553669 & $\mathrm{~T}$ & 0.027 & $4.9 \times 10^{-5}$ & 0.021 & $8.1 \times 10^{-6}$ & $6.7 \times 10^{-10}$ & $H L A-D R A$ \\
\hline rs1936797 & 6 & 127474350 & A & 0.022 & $2.8 \times 10^{-3}$ & 0.022 & $9.9 \times 10^{-7}$ & $6.7 \times 10^{-9}$ & RSPO3 \\
\hline rs13244268 & 7 & 72549779 & $T$ & 0.054 & $2.6 \times 10^{-8}$ & -0.045 & $1.3 \times 10^{-9}$ & $1.2 \times 10^{-13}$ & $B A Z 1 B$ \\
\hline rs9987289 & 8 & 9220768 & A & -0.079 & $2.1 \times 10^{-12}$ & -0.083 & $6.4 \times 10^{-25}$ & $1.2 \times 10^{-39}$ & PPP1R3B \\
\hline rs4871137 & 8 & 121937732 & $\mathrm{~T}$ & -0.021 & $2.2 \times 10^{-3}$ & -0.026 & $5.6 \times 10^{-6}$ & $3.3 \times 10^{-8}$ & SNTB1 \\
\hline rs10761731 & 10 & 64697616 & $A$ & 0.023 & $2.7 \times 10^{-4}$ & -0.025 & $2.5 \times 10^{-7}$ & $2.2 \times 10^{-8}$ & JMJDIC \\
\hline rs174546 & 11 & 61326406 & $T$ & -0.017 & $1.2 \times 10^{-2}$ & -0.048 & $2.6 \times 10^{-22}$ & $1.6 \times 10^{-24}$ & FADS1 \\
\hline rs1077834 & 15 & 56510771 & $\mathrm{~T}$ & -0.016 & $4.0 \times 10^{-2}$ & -0.114 & $9.6 \times 10^{-84}$ & $2.5 \times 10^{-87}$ & LIPC \\
\hline rs1558902 & 16 & 52361075 & A & 0.032 & $2.0 \times 10^{-6}$ & -0.021 & $4.6 \times 10^{-6}$ & $5.0 \times 10^{-9}$ & FTO \\
\hline rs711752 & 16 & 55553712 & $A$ & 0.016 & $1.8 \times 10^{-2}$ & 0.192 & $2.1 \times 10^{-297}$ & $4.3 \times 10^{-308}$ & CETP \\
\hline rs17688076 & 16 & 66843928 & A & 0.019 & $4.9 \times 10^{-2}$ & 0.070 & $3.9 \times 10^{-22}$ & $1.8 \times 10^{-23}$ & LYPLA3 \\
\hline rs11874381 & 18 & 45457406 & A & 0.013 & $4.9 \times 10^{-2}$ & 0.038 & $1.2 \times 10^{-14}$ & $1.0 \times 10^{-15}$ & $L I P G$ \\
\hline rs12967135 & 18 & 56000003 & A & 0.029 & $1.2 \times 10^{-4}$ & -0.036 & $6.6 \times 10^{-9}$ & $4.3 \times 10^{-10}$ & MC4R \\
\hline rs4420638 & 19 & 50114786 & A & 0.240 & $1.0 \times 10^{-129}$ & 0.071 & $4.4 \times 10^{-21}$ & $2 \times 10^{-164}$ & $A P O C 1$ \\
\hline rs1800961 & 20 & 42475778 & $T$ & -0.120 & $2.4 \times 10^{-11}$ & -0.129 & $1.1 \times 10^{-15}$ & $3.9 \times 10^{-28}$ & HNF4A \\
\hline rs6065906 & 20 & 43987422 & $T$ & 0.036 & $5.9 \times 10^{-6}$ & 0.058 & $1.9 \times 10^{-22}$ & $5.1 \times 10^{-29}$ & PLTP \\
\hline
\end{tabular}

Abbreviations: SNP single nucleotide polymorphism

For both CRP and the lipid phenotype, the effect estimates are according to the original GWAS

Chromosome and position are in NCBI genome build 36

$\beta$ coefficient for CRP represents 1-unit change in the natural log-transformed CRP ( $\mathrm{mg} / \mathrm{L}$ ) per copy increment in the coded allele

Beta coefficient for HDL-cholesterol represents 1-unit change in the standardized HDL-cholesterol levels per copy increment in the coded allele

C7orf50 were novel pleiotropic loci for both CRP and total cholesterol.

\section{Replication of the novel pleiotropic loci}

In total, we sought replication for 36 potential novel SNPs for CRP in 17,743 genotyped individuals from three independent cohort studies. Using a Bonferroni corrected threshold for multiple testing $(0.05 / 36=1.4 \times$ $10^{-3}$ ), three SNPs remained significantly associated with CRP levels when we performed replication analysis (Additional file 1: Table S1). These variants included the SNPs rs10435719 in CTSB/FDFT1 $\left(P_{\text {replication }}=2.6 \times 10^{-5}\right)$, rs1558902 near FTO $\left(P_{\text {replication }}=2.7 \times 10^{-5}\right)$ and $\mathrm{rs} 7621025$ near STAG1 $\left(P_{\text {replication }}=1.4 \times 10^{-3}\right)$.

We aimed replication for 23 potential novel SNPs for lipids (4 for LDL-cholesterol, 7 for HDL-cholesterol, 9 for triglycerides and 3 for total cholesterol) in an in silico analysis including 93,982 individuals. We could significantly replicate 2 variants for LDL-cholesterol (HNF4A and RASIP1), three for HDL-cholesterol (C1orf172, RSPO3 and STAG1), one for triglycerides (CTSB) and one for total cholesterol (C7orf50) (Additional file 1: Table S2).

\section{Expression Quantitative Trait Loci (eQTL)}

To annotate the effect of the replicated pleiotropic variants to the expression level of nearby genes, we investigated the association between the pleiotropic variants and gene expression levels in three different tissues relevant to CRP and lipids by use of large publicly available datasets: whole blood $(N=5311)$ [9], liver $(N=427$ [10] and 266 [11]) and adipose tissue [12] $(N=111)$. For the replicated pleiotropic variant rs10435719 near CTSB and FDFT1, we observed significant associations in whole blood with expression levels of two genes: CTSB itself $\left(P=1.67 \times 10^{-6}\right)$, and FDFT1 $\left(P=1.10 \times 10^{-96}\right)$. In addition, the SNP rs7621025 near STAG1 and PCCB was strongly associated with expression of the gene $P C C B$ in whole blood $\left(P=1.1 \times 10^{-40}\right)$. No eQTL effect was observed in the liver and adipose tissue.

\section{Discussion}

We identified fifty potential pleiotropic SNPs which affect both CRP and lipid levels, of which we replicated three novel CRP variants: rs10435719 (CTSB/FDFT1), rs7621025 (STAG1/PCCB) and rs1558902 (FTO). In silico expression analyses suggested a role for 
Table 3 Results of Bivariate GWAS Analyses for C-Reactive Protein and Triglycerides Levels

\begin{tabular}{|c|c|c|c|c|c|c|c|c|c|}
\hline \multirow[t]{2}{*}{ SNP } & \multirow[t]{2}{*}{ Chromosome } & \multirow[t]{2}{*}{ Position } & \multirow{2}{*}{$\begin{array}{l}\text { Effect } \\
\text { Allele }\end{array}$} & \multicolumn{2}{|c|}{ C-reactive protein } & \multicolumn{2}{|c|}{ Triglycerides } & \multirow{2}{*}{$\begin{array}{l}\text { Pleiotropy } \\
\text { significance }\end{array}$} & \multirow[t]{2}{*}{ Gene } \\
\hline & & & & $\overline{\text { Beta }}$ & P-value & Beta & P-value & & \\
\hline rs4660808 & 1 & 39791096 & $\mathrm{~T}$ & 0.046 & $8.6 \times 10^{-10}$ & 0.028 & $3.1 \times 10^{-7}$ & $2.2 \times 10^{-13}$ & PABPC4 \\
\hline rs11208722 & 1 & 65943589 & $A$ & -0.083 & $1.2 \times 10^{-32}$ & 0.012 & 0.02 & $8.1 \times 10^{-36}$ & LEPR \\
\hline rs1127311 & 1 & 152823287 & $A$ & -0.031 & $9.3 \times 10^{-7}$ & 0.012 & $5.5 \times 10^{-3}$ & $6.4 \times 10^{-9}$ & $A D A R$ \\
\hline rs12755606 & 1 & 157936960 & C & -0.153 & $3.0 \times 10^{-112}$ & 0.012 & 0.01 & $4.0 \times 10^{-120}$ & $C R P$ \\
\hline rs1260326 & 2 & 27584444 & $\mathrm{~T}$ & 0.089 & $1.7 \times 10^{-42}$ & 0.116 & $5.7 \times 10^{-133}$ & $4.4 \times 10^{-151}$ & GCKR \\
\hline rs13409360 & 2 & 113554573 & $A$ & 0.048 & $1.3 \times 10^{-12}$ & -0.013 & $8.6 \times 10^{-3}$ & $5.3 \times 10^{-15}$ & ILIF10 \\
\hline rs645040 & 3 & 137409312 & $\mathrm{~T}$ & -0.023 & $2.5 \times 10^{-3}$ & 0.030 & $2.5 \times 10^{-8}$ & $4.6 \times 10^{-11}$ & MSL2L1 \\
\hline rs2524163 & 6 & 31367558 & $\mathrm{~T}$ & 0.025 & $1.5 \times 10^{-4}$ & 0.027 & $1.7 \times 10^{-8}$ & $7.9 \times 10^{-10}$ & HLA-C \\
\hline rs9987289 & 8 & 9220768 & $A$ & -0.079 & $2.1 \times 10^{-12}$ & 0.020 & 0.02 & $2.9 \times 10^{-14}$ & PPP1R3B \\
\hline rs10435719 & 8 & 11814313 & $\mathrm{~T}$ & 0.026 & $7.6 \times 10^{-5}$ & -0.022 & $4.1 \times 10^{-6}$ & $2.0 \times 10^{-10}$ & CTSB \\
\hline rs1441759 & 8 & 19909843 & $C$ & 0.11 & $3.3 \times 10^{-4}$ & 0.125 & $2.1 \times 10^{-8}$ & $2.0 \times 10^{-9}$ & $L P L$ \\
\hline rs10832027 & 11 & 13313759 & A & 0.032 & $8.5 \times 10^{-7}$ & 0.020 & $1.1 \times 10^{-4}$ & $9.4 \times 10^{-9}$ & ARNTL \\
\hline rs174546 & 11 & 61326406 & $T$ & -0.017 & 0.01 & 0.048 & $5.4 \times 10^{-24}$ & $5.2 \times 10^{-27}$ & FADS1 \\
\hline rs2686555 & 12 & 119579555 & A & -0.059 & $1.7 \times 10^{-19}$ & 0.010 & 0.03 & $1.6 \times 10^{-21}$ & CABP1 \\
\hline rs11508026 & 16 & 55556829 & $\mathrm{~T}$ & 0.014 & 0.03 & -0.038 & $1.3 \times 10^{-12}$ & $3.1 \times 10^{-14}$ & CETP \\
\hline rs571312 & 18 & 55990749 & $A$ & 0.033 & $3.5 \times 10^{-5}$ & 0.026 & $1.2 \times 10^{-5}$ & $2.8 \times 10^{-8}$ & MC4R \\
\hline rs10401969 & 19 & 19268718 & $T$ & -0.031 & 0.02 & 0.112 & $1.6 \times 10^{-29}$ & $1.6 \times 10^{-32}$ & SF4 \\
\hline rs1688043 & 19 & 40245181 & $T$ & -0.038 & $2.4 \times 10^{-3}$ & 0.037 & $1.2 \times 10^{-5}$ & $4.1 \times 10^{-8}$ & HPN \\
\hline rs4420638 & 19 & 50114786 & A & 0.24 & $1.0 \times 10^{-129}$ & -0.068 & $5.4 \times 10^{-22}$ & $1.7 \times 10^{-171}$ & $A P O C 1$ \\
\hline rs4465830 & 20 & 44018827 & $A$ & 0.036 & $7.0 \times 10^{-6}$ & -0.050 & $2.0 \times 10^{-17}$ & $2.0 \times 10^{-24}$ & ZNF335/PLTP \\
\hline rs2277844 & 22 & 36907461 & $A$ & -0.018 & $5.7 \times 10^{-3}$ & 0.025 & $1.5 \times 10^{-7}$ & $9.2 \times 10^{-10}$ & PLA2G6 \\
\hline
\end{tabular}

Abbreviations: SNP single nucleotide polymorphism

For both CRP and the lipid phenotype, the effect estimates are according to the original GWAS

Chromosome and position are in NCBI genome build 36

$\beta$ coefficient for CRP represents 1-unit change in the natural log-transformed CRP (mg/L) per copy increment in the coded allele

Beta coefficient for triglycerides represents 1-unit change in the standardized triglyceride levels per copy increment in the coded allele

rs10435719 in the gene expression of both CTSB and FDFT1 and rs7621025 appeared to have an effect on the gene expression of $P C C B$.

The locus harboring rs10435719 near CTSB and FDFT1 that was identified for CRP in our study has previously been identified for triglycerides in the joint analysis of the Global Lipids Genetics Consortium combining GWAS data with Metabochip association results [13]. We observed a significant effect of rs10435719 on the expression of both CTSB and FDFT1. The effect of the CRP increasing allele $(\mathrm{T})$ was weakly associated with a decrease in the expression of CTSB, whilst we observed a strong association of the T-allele with an increase of FDFT1 gene expression. FDFT1 encodes the enzyme squalene synthase which is involved in the cholesterol biosynthesis [14]. Apart from lipids, FDFT1 has been identified in a GWAS on fatty liver disease [15]. Squalene Synthase Inhibitors (SSI) have been developed and are successful in the reduction of cholesterol levels as well as CRP levels [16]. This pleiotropic effect of cholesterol synthesis blockers on both lipid levels and inflammation is thought to be the consequence of altered isoprenoids levels that may activate pro-inflammatory pathways [17]. The observation that the CRP increasing allele is associated with an increase in FDFT1 gene expression suggests an effect of rs10435719 on serum CRP through FDFT1. However, we searched in large databases to identify robust eQTL effects of the novel variants. Therefore, we were unable to test the association between the expression and CRP and we cannot draw a firm conclusion on the causal effect of the gene expression in the association between the genetic variant and CRP.

We identified the SNP rs7621025 (STAG1/PCCB) as a pleiotropic variant for HDL-cholesterol and CRP. We confirmed the effect of rs7621025 on serum CRP in an independent set of individuals and this genomic region has been identified in a GWAS of lipids [13]. The SNP rs7621025 is located within STAG1, but has a strong effect on the expression of $P C C B$, located $\pm 300 \mathrm{~kb}$ downstream of rs7621025 on chromosome 3. $P C C B$ has been identified in a GWAS of the protein fibrinogen, an acute 
Table 4 Results of Bivariate GWAS Analyses for C-Reactive Protein and Total Cholesterol Levels

\begin{tabular}{|c|c|c|c|c|c|c|c|c|c|}
\hline \multirow[t]{2}{*}{ SNP } & \multirow[t]{2}{*}{ Chromosome } & \multirow[t]{2}{*}{ Position } & \multirow{2}{*}{$\begin{array}{l}\text { Effect } \\
\text { Allele }\end{array}$} & \multicolumn{2}{|c|}{ C-reactive protein } & \multicolumn{2}{|c|}{ Total cholesterol } & \multirow{2}{*}{$\begin{array}{l}\text { Pleiotropy } \\
\text { significance }\end{array}$} & \multirow[t]{2}{*}{ Gene } \\
\hline & & & & Beta & P-value & Beta & P-value & & \\
\hline rs469772 & 1 & 91302893 & $\mathrm{~T}$ & -0.042 & $1.6 \times 10^{-7}$ & -0.020 & $1.5 \times 10^{-3}$ & $1.5 \times 10^{-8}$ & ZNF644 \\
\hline rs629301 & 1 & 109619829 & $\mathrm{~T}$ & -0.017 & $2.8 \times 10^{-2}$ & 0.149 & $5.8 \times 10^{-131}$ & $5.7 \times 10^{-132}$ & CELSR2 \\
\hline rs17597773 & 1 & 219121384 & $C$ & 0.020 & $7.5 \times 10^{-3}$ & -0.031 & $7.1 \times 10^{-8}$ & $6.6 \times 10^{-9}$ & $H L X$ \\
\hline rs661955 & 1 & 232909479 & $C$ & -0.021 & $1.7 \times 10^{-3}$ & 0.036 & $1.0 \times 10^{-12}$ & $2.2 \times 10^{-14}$ & IRF2BP2 \\
\hline rs1260326 & 2 & 27584444 & $\mathrm{~T}$ & 0.089 & $1.7 \times 10^{-42}$ & 0.055 & $7.3 \times 10^{-27}$ & $2.6 \times 10^{-63}$ & GCKR \\
\hline rs4148191 & 2 & 43896408 & A & -0.050 & $2.5 \times 10^{-4}$ & -0.054 & $1.1 \times 10^{-6}$ & $3.7 \times 10^{-09}$ & $A B C G 5$ \\
\hline rs6734238 & 2 & 113557501 & A & -0.047 & $4.8 \times 10^{-13}$ & 0.023 & $1.2 \times 10^{-5}$ & $5.8 \times 10^{-17}$ & ILIF10 \\
\hline rs4703642 & 5 & 74297918 & A & 0.018 & $3.0 \times 10^{-3}$ & -0.033 & $2.0 \times 10^{-11}$ & $7.3 \times 10^{-13}$ & GCNT4 \\
\hline rs577272 & 6 & 31945942 & A & 0.020 & $1.1 \times 10^{-3}$ & 0.026 & $2.3 \times 10^{-7}$ & $1.6 \times 10^{-8}$ & SLC44A4 \\
\hline rs2858310 & 6 & 32776301 & A & 0.026 & $8.7 \times 10^{-5}$ & 0.033 & $3.3 \times 10^{-10}$ & $3.8 \times 10^{-12}$ & HLA-DQB1 \\
\hline rs3822857 & 6 & 116420624 & C & -0.032 & $2.7 \times 10^{-6}$ & -0.033 & $4.7 \times 10^{-9}$ & $2.1 \times 10^{-12}$ & FRK \\
\hline rs6951245 & 7 & 1024719 & A & 0.03 & $5.5 \times 10^{-4}$ & 0.037 & $6.1 \times 10^{-8}$ & $2.6 \times 10^{-9}$ & C7orf50 \\
\hline rs2126259 & 8 & 9222556 & $\mathrm{~T}$ & -0.072 & $5.7 \times 10^{-12}$ & -0.085 & $9.0 \times 10^{-24}$ & $1.4 \times 10^{-31}$ & PPP1R3B \\
\hline rs11220463 & 11 & 125753421 & A & 0.032 & $2.8 \times 10^{-3}$ & -0.057 & $2.1 \times 10^{-11}$ & $7.3 \times 10^{-13}$ & STSGAL4 \\
\hline rs1183910 & 12 & 119905190 & A & -0.151 & $4.6 \times 10^{-113}$ & 0.040 & $5.2 \times 10^{-14}$ & $8.2 \times 10^{-128}$ & HNF1A \\
\hline rs340025 & 15 & 58695599 & $\mathrm{~T}$ & -0.036 & $8.3 \times 10^{-9}$ & 0.015 & $2.4 \times 10^{-3}$ & $2.5 \times 10^{-10}$ & RORA \\
\hline rs1529711 & 19 & 10884434 & $\mathrm{~T}$ & 0.030 & $8.4 \times 10^{-4}$ & 0.038 & $6.3 \times 10^{-7}$ & $3.4 \times 10^{-8}$ & CARM1 \\
\hline rs2228603 & 19 & 19190924 & $\mathrm{~T}$ & 0.036 & $2.9 \times 10^{-3}$ & -0.118 & $4.3 \times 10^{-34}$ & $1.1 \times 10^{-35}$ & NCAN \\
\hline rs4420638 & 19 & 50114786 & A & 0.240 & $1.0 \times 10^{-129}$ & -0.184 & $5.2 \times 10^{-111}$ & $3.8 \times 10^{-249}$ & APOC1 \\
\hline rs1800961 & 20 & 42475778 & $\mathrm{~T}$ & -0.120 & $2.4 \times 10^{-11}$ & -0.118 & $5.7 \times 10^{-13}$ & $1.0 \times 10^{-20}$ & HNF4A \\
\hline
\end{tabular}

Abbreviations: SNP single nucleotide polymorphism

For both CRP and the lipid phenotype, the effect estimates are according to the original GWAS

Chromosome and position are in NCBI genome build 36

Beta coefficient for total cholesterol represents 1-unit change in the standardized total cholesterol levels per copy increment in the coded allele

$\beta$ coefficient for CRP represents 1-unit change in the natural log-transformed CRP ( $\mathrm{mg} / \mathrm{L}$ ) per copy increment in the coded allele

phase response protein sharing many genes with CRP [18]. Our results provide further evidence that the $P C C B$ gene is involved in inflammation.

We identified the FTO gene as a pleiotropic locus for CRP and HDL-cholesterol. The A allele of rs1558902 was associated with an increase of CRP and a decrease in HDL cholesterol. In several GWAS on BMI, the A allele of rs1558902 was also associated with an increase in BMI [19, 20]. Previous studies have highlighted the causal effect of obesity on inflammation [21], and the effect directions are consistent with mediation of both the association with CRP and HDL-cholesterol by BMI. We have previously shown that the effect of FTO on CRP is indeed mediated through BMI [22]. Further research is needed to demonstrate whether this is also true for HDL-cholesterol. Our results provide further evidence for the role of obesity in inflammation and highlight the pleiotropic effects of the FTO locus on both chronic inflammation and lipid metabolism.

Genetic pleiotropy can be divided in biological and mediated pleiotropy [4]. In biological pleiotropy, the effect of the pleiotropic variant on two or more phenotypes is independent. In mediated pleiotropy, one phenotype mediates the association between the genetic variant and the second phenotype. Both biological and mediated pleiotropic effects may occur for CRP and lipids [23]. In the current study, we did not disentangle the different subtypes of pleiotropy. Moreover, we observed pleiotropic variants with an opposite direction of effect than expected based on the phenotypical correlation in observational epidemiological studies. In biological pleiotropy, opposite directions of effect may occur. As an example, although CRP and LDLcholesterol are positively associated in observational epidemiological studies, the A-allele of the SNP rs1183910 (HNF1A) is associated with lower CRP levels but higher LDL-cholesterol. Opposite direction of effects are often seen in genetic studies and highlight the complex interplay between correlated phenotypes, in our study CRP and lipids [20]. We did not disentangle the different subtypes of pleiotropy, which is a limitation of the current study.

Our study has certain strengths. We add to previous studies showing that the multivariate method we applied can be effectively utilized to identify potential novel and pleiotropic loci. This method only requires GWAS 
summary data instead of individual level data from all participating cohorts. Thanks to close collaboration between studies across the world, researchers have performed large GWAS meta-analyses for a vast amount of phenotypes and this data is available for further research. Second, we used the largest GWAS meta-analyses that have so far been done on CRP and lipid levels to identify pleiotropic genetic loci. By doing so, we enhanced the statistical power to detect these loci considerably. Third, we provided robust evidence for three novel CRP loci by replication in an independent sample of genotyped individuals. A limitation of the bivariate meta-analysis is that very strong signals in one of the individual traits may overshadow the weak association with the other phenotype. We set a criterion for the univariate $\mathrm{p}$-values $<0.05$ to minimize the chance of false positive findings. In many instances the effect of the pleiotropic loci on CRP or lipids is very small. We did not replicate all our pleiotropic loci. This could be due to lack of power in the replication. In concordance, we replicated a larger proportion of the lipid variants in the larger lipid replication sample compared to CRP. Also, variants closer to significance did replicate in the replication study of both CRP and lipids. Also, several variants had substantial heterogeneity $\mathrm{I}^{2}$ in the replication which lowers the power for replication. Furthermore, the replication sample size was for some variants smaller than 17,743 due to absence of the variants in one or more of the replication studies. However, we cannot rule out the possibility that bivariate $\mathrm{p}$-values are driven by strong associations with one of the phenotypes and produce false positive results. In addition, for the replication of the lipid variants, we used the Metabochip results from the GLGC. Several variants selected for replication were not present on the Metabochip. Although we selected the best available proxy SNP for replication, variants in moderate LD may have limited power for replication. The method used in the current manuscript to prioritize variants with pleiotropic effects among inflammation and cholesterol are hypothesis generating and further functional work regarding the role of the identified variants in cholesterol metabolism and inflammation is necessary.

\section{Conclusions}

Our results provide evidence for substantial overlap in genetic susceptibility for chronic inflammation and lipid metabolism. In addition, through bivariate genome-wide association studies and replication in an independent sample of individuals we could identify novel genes for CRP.

\section{Methods}

The present study includes three stages. First, we performed a bivariate GWAS combining published GWAS data on CRP and lipids to identify pleiotropic variants for CRP and lipids. In a second step, we sought replication of novel associations in independent samples of genotyped individuals. Finally, we carried out functional analyses in a third step to point out potential underlying transcriptional mechanisms.

We used the data from the largest published GWAS on CRP as well as the publically available GWAS on lipids from GLGC to explore the genetic pleiotropy between inflammation and lipids [2, 3]. We combined summary association test statistics from the CRP GWAS separately with the GWAS on HDL-cholesterol, LDLcholesterol, triglycerides and total cholesterol. The CRP GWAS meta-analysis included 65,000 individuals from 15 different studies in the discovery panel and after replication, 18 loci were genome-wide significantly associated with serum CRP level [3]. The lipids GWAS comprised 100,184 individuals for total cholesterol, 95,454 for LDL-cholesterol, 99,900 for HDL-cholesterol and 96,598 for triglycerides across 46 studies. The lipid GWAS identified a total of 95 lipid loci $(52$ for total cholesterol, 37 for LDL-cholesterol, 47 for HDLcholesterol and 32 for triglycerides) [2]. The CRP and lipids GWAS used HapMap imputed data (build 36). All studies that contributed genotype data to the CRP GWAS also contributed data to the lipids GWAS. We ensured that effect alleles were harmonized across the two GWAS before applying the bivariate GWAS method. Overall, 2,501,549 common Single Nucleotide Polymorphisms (SNPs) were tested for their association with CRP and total cholesterol, 2,501,711 with CRP and triglycerides, 2,501,543 with CRP and HDL-cholesterol and 2,501,749 with CRP and LDL-cholesterol. An aggregated p-value was calculated using the method described below.

\section{Bivariate genome-wide association study}

To better understand the shared biology of CRP and lipids by further identifying shared genes between CRP and lipids, we aimed to increase power by combining the summary statistics from the CRP and lipid GWAS. We chose to use a recently introduced method that performs bivariate GWAS allowing for mixed directions of effect. The method combines summary statistics ( $Z$ test statistics) from univariate GWAS of CRP pairing with the summary statistics of each univariate GWAS metaanalysis of lipid phenotypes, using an empirical-weighted linear-combined test statistics (eLC), implemented in a $\mathrm{C}++$ eLX package. We have recently used this method in the identification of pleiotropic genes for menopause and menarche and the details of the method are presented elsewhere. [8, 24]. eLC allows having opposite direction of effect on the combined phenotypes, which is common between CRP and cholesterol phenotypes $[2,3]$. 
Briefly, eLC directly combines correlated Z test statistics (calculated as $\beta / S E$ derived from the original GWAS) obtained from univariate GWAS meta-analyses with a weighted sum of univariate test statistics to empirically maximize the overall association signals and also to account for the phenotypical correlations among CRP and lipids. Our eLC approach is expressed as

$$
S_{e L C}=\sum_{1}^{k}\left[\max \left(\left|T_{k}\right|, c\right)^{*}\left|T_{k}\right|\right]
$$

where $T_{k}$ is a matrix of $K$ statistics for $K$ phenotypes (for bivariate, $\mathrm{K}$ is equal to 2 ) and $\mathrm{c}$ is a given nonnegative constant. The optimal weighting is estimated empirically using the Monte Carlo Simulation [25] and the bona-fide p-values for eLC test statistics are calculated through permutation. The sample covariance matrix of the test statistics of all SNPs from the univariate GWAS analyses is used as an approximation of the variancecovariance matrix $\Sigma$ of univariate test statistics. $\Sigma$ :

$$
\left[\begin{array}{cc}
\operatorname{Var}\left(Z_{1}\right) & \operatorname{Cov}\left(Z_{1},, Z_{2}\right) \\
\operatorname{Cov}\left(Z_{1},, Z_{2}\right) & \operatorname{Var}\left(Z_{2}\right)
\end{array}\right]
$$

where $Z_{1}$ and $Z_{2}$ consist of unbiased univariate test statistics of all the SNPs for the two traits on genomewide scale for the first $\left(Z_{1}\right)$ and second $\left(Z_{2}\right)$ trait. The null hypothesis in the bivariate analysis is $\beta \_1=0$ AND $\beta \_2=0$; the $H 1$ is $\beta \_1$ not equal to 0 or $\beta \_2$ not equal to 0 . The results were considered genome-wide significant when (1) the bivariate p-values were $<5 \times 10^{-8}$ and (2) the bivariate $\mathrm{p}$-value was at least one order of magnitude lower than both individual trait p-values and (3) when the individual trait $\mathrm{p}$-values were at least nominally significant ( $\mathrm{p}$-value $<0.05$ ). When multiple SNPs were significant in a locus, the SNP with the lowest p-value was chosen for replication. The eLC method is implemented in eLX package using $\mathrm{C}++$ (see Weblinks).

\section{Replication study}

The bivariate GWAS resulted in three possible scenarios. First, the pleiotropic variant or the locus harboring the pleiotropic variant (defined as $\pm 500 \mathrm{MB}$ of the pleiotropic SNP) was genome-wide significant in both the primary univariate GWAS of CRP and the lipid trait. Second, the pleiotropic signal was significant in either the CRP or the lipid univariate GWAS. Third, the pleiotropic signal was neither genome-wide significant in the CRP nor in the lipid GWAS. Per definition, a variant is considered pleiotropic when there is robust evidence for an association with two or more phenotypes. Therefore, we only selected the variants that were not genome-wide significant in the primary univariate GWAS for replication in an independent sample of genotyped samples. We intended to replicate the novel associations with
CRP levels in three cohort studies that did not contribute to the original CRP GWAS. The independent cohorts were the second $(n=1943)$ and third $(n=2962)$ cohort of the Rotterdam Study and the LifeLines cohort study ( $n=12,838$; Additional file 1) [6, 7, 26, 27]. The total sample size for the replication of potentially novel CRP variants comprised 17,743 individuals. In an attempt to replicate the potential novel lipid variants, we performed an in silico replication in the publicly available association results from the participants of the GLGC that did not contribute to the original lipids GWAS we used for the pleiotropy analysis. This replication set comprises 93,982 individuals genotyped using the Metabochip array $[13,28]$. For the SNPs that were not available on the Metabochip, we selected the best available proxy SNP on the Metabochip for replication $\left(r^{2}>0.5\right)$. We used a Bonferroni corrected p-value of 0.05 divided by the number of SNPs tested for replication as a threshold of significance in the replication study.

\section{Ethics, consent and permissions}

All participants of the Rotterdam and Lifelines study provided written informed consent.

\section{Expression Quantitative Trait Loci (eQTL)}

In an attempt to annotate the pleiotropic variants to a pleiotropic gene, we searched in tissues related to lipids and inflammation for eQTL effects of the pleiotropic variants or reasonable proxy variants $\left(r^{2}>0.80\right)$.

The eQTL analyses in whole blood comprised 5311 individuals from seven studies in the discovery setting with both genetic and gene expression data available [9]. The discovery meta-analysis including the seven studies (EGCUT, InCHIANTI, Rotterdam Study, Fehrmann, HVH, SHIP-TREND and DILGOM). Results are publicly available (access URL: http://genenetwork.nl/bloodeqtlbrowser/). eQTLs were deemed cis when the distance between the SNP and the midpoint of the RNA probe was $<250 \mathrm{~kb}$. We only considered a significant eQTL effect of the pleiotropic SNP when the p-value exceeded the FDR corrected threshold for multiple testing.

We searched for liver eQTL effects by use of the eQTL browser provided by the university of Chicago (access URL: http://eqtl.uchicago.edu/cgi-bin/gbrowse/ eqtl/). The liver tissue dataset by Schadt et al. comprised 427 individuals from European ancestry with liver specific gene expression and genotyping data available [10]. An eQTL was deemed cis when the SNP was within $1 \mathrm{Mb}$ of the annotated start or stop site of the corresponding structural gene. The authors used an FDR correction of $10 \%$ for a significant association. The dataset by Innocenti et al. comprised 266 individuals from 2 different studies. Cis eQTL was defined as $<250 \mathrm{~kb}$ from 
the gene transcription start site and the FDR for significant association was set to $5 \%$ [11].

We used the GTEx adipose tissue dataset (access URL: http://www.gtexportal.org/home/eqtls/tissue?tissueName=Adipose_Subcutaneous) to search for potential eQTLs in adipose tissue. The dataset consisted of 111 individuals with both gene expression and genotype data available [12] Cis radius was defined as $+/-1 \mathrm{mb}$ from transcription start site. A eQTL was deemed significant when the FDR q-value $<=5 \%$.

\section{Additional file}

Additional file 1: Study-specific Methods Section for the Replication Section. Table S1. Replication Results for C-Reactive Protein. Table S2. Replication Results for lipids. Table S3. Proxy variants for the Single Nucleotide Polymophisms not Available on the Metabochip Array. (DOCX $30 \mathrm{~kb}$ )

\section{Abbreviations}

CRP: C-reactive protein; GWAS: genome-wide association study.

\section{Acknowledgments}

Data on lipid traits have been contributed by GLGC investigators and have been downloaded from http://www.broadinstitute.org/mpg/pubs/lipids2010/. We thank Behrooz Z. Alizadeh, Annemieke Boesjes, Marcel Bruinenberg, Noortje Festen, Pim van der Harst, llja Nolte, Lude Franke, Mitra Valimohammadi for their help in creating the GWAS database, and Rob Bieringa, Joost Keers, René Oostergo, Rosalie Visser, Judith Vonk for their work related to data-collection and validation. The authors are grateful to the study participants, the staff from the LifeLines Cohort Study and Medical Biobank Northern Netherlands, and the participating general practitioners and pharmacists. Researchers interested in using the LifeLines data must obtain approval for a specific analysis plan from the scientific board of LifeLines to obtain access to the data. Researchers using the data are required to follow the terms of a signed agreement containing a number of clauses designed to ensure protection of privacy and compliance with relevant laws. We thank Pascal Arp, Mila Jhamai, Marijn Verkerk, Lizbeth Herrera and Marjolein Peters for their help in creating the GWAS database in the Rotterdam Study, and Karol Estrada, Yurii Aulchenko and Carolina Medina-Gomez for the creation and analysis of imputed data. The authors are grateful to the study participants, the staff from the Rotterdam Study and the participating general practitioners and pharmacists.

\section{Authors' contributions}

SL carried out the pleiotropy analyses, the replication analyses in the Rotterdam cohorts and drafted the manuscript. AV analyzed the LifeLines cohort data and contributed to the manuscript writing. YHH developed the pleiotropy method, created the software and helped interpreting the results. RS, AGU, AH, BZA and OHF designed the study and critically reviewed the manuscript. AD designed the study, helped interpreting the results and critically reviewed the manuscript. All authors read and approved the final manuscript.

\section{Competing interests}

The authors declare that they have no competing interests.

\section{Sources of funding}

The LifeLines Cohort Study is supported by the Netherlands Organization of Scientific Research NWO (grant 175.010.2007.006), the Economic Structure Enhancing Fund (FES) of the Dutch government, the Ministry of Economic Affairs, the Ministry of Education, Culture and Science, the Ministry for Health, Welfare and Sports, the Northern Netherlands Collaboration of Provinces (SNN), the Province of Groningen, University Medical Center Groningen, the University of Groningen, Dutch Kidney Foundation and Dutch Diabetes Research Foundation. The authors wish to acknowledge the services of the
LifeLines Cohort Study, the contributing research centers delivering data to LifeLines, and all the study participants.

The generation and management of GWAS genotype data for the Rotterdam Study is supported by the Netherlands Organisation of Scientific Research NWO Investments (nr. 175.010.2005.011, 911-03-012). This study is funded by the Research Institute for Diseases in the Elderly (014-93-015; RIDE2), the Netherlands Genomics Initiative (NGI)/Netherlands Organisation for Scientific Research (NWO) project nr. 050-060-810. The Rotterdam Study is funded by Erasmus Medical Center and Erasmus University, Rotterdam, Netherlands Organization for the Health Research and Development (ZonMw), the Research Institute for Diseases in the Elderly (RIDE), the Ministry of Education, Culture and Science, the Ministry for Health, Welfare and Sports, the European Commission (DG XII), and the Municipality of Rotterdam.

\section{Weblinks}

eLc methods in eLX package: https://sites.google.com/site/ multivariateyihsianghsu/.

\section{Author details}

'Department of Epidemiology, Erasmus University Medical Center, PO Box 20403000CA Rotterdam, The Netherlands. ${ }^{2}$ Department of Epidemiology, University of Groningen, University Medical Center Groningen, Groningen, The Netherlands. ${ }^{3} \mathrm{Hebrew}$ SeniorLife Institute for Aging Research and Harvard Medical School, Boston, MA, USA. ${ }^{4}$ Molecular and Integrative Physiological Sciences Program, Harvard School of Public Health, Boston, MA, USA. ${ }^{5}$ Framingham Heart Study, Framingham, MA, USA. ${ }^{6}$ Department of Internal Medicine, Erasmus University Medical Center, Rotterdam, The Netherlands.

Received: 1 September 2015 Accepted: 6 May 2016

Published online: 10 June 2016

\section{References}

1. Deloukas P, Kanoni S, Willenborg C, Farrall M, Assimes TL, Thompson JR, Ingelsson E, Saleheen D, Erdmann J, Goldstein BA. Large-scale association analysis identifies new risk loci for coronary artery disease. Nat Genet. 2012. doi:10.1038/ng.2480.

2. Teslovich TM, Musunuru K, Smith AV, Edmondson AC, Stylianou IM, Koseki M, Pirruccello JP, Ripatti S, Chasman DI, Willer CJ. Biological, clinical and population relevance of 95 loci for blood lipids. Nature. 2010;466(7307):707-13.

3. Dehghan A, Dupuis J, Barbalic M, Bis JC, Eiriksdottir G, Lu C, Pellikka N, Wallaschofski H, Kettunen J, Henneman P. Meta-analysis of genome-wide association studies in $>80000$ subjects identifies multiple loci for C-reactive protein levels. Circulation. 2011;123(7):731-8.

4. Solovieff N, Cotsapas C, Lee PH, Purcell SM, Smoller JW. Pleiotropy in complex traits: challenges and strategies. Nat Rev Genet. 2013;14:483-95.

5. Albert MA, Danielson E, Rifai N, Ridker PM. Effect of statin therapy on C-reactive protein levels. JAMA. 2001;286(1):64-70

6. Ridker PM, Danielson E, Fonseca FAH, Genest J, Gotto Jr AM, Kastelein JJP, Koenig W, Libby P, Lorenzatti AJ, MacFadyen JG. Reduction in C-reactive protein and LDL cholesterol and cardiovascular event rates after initiation of rosuvastatin: a prospective study of the JUPITER trial. Lancet. 2009;373(9670):1175-82.

7. Jain MK, Ridker PM. Anti-inflammatory effects of statins: clinical evidence and basic mechanisms. Nat Rev Drug Discov. 2005;4:977-87.

8. Perry JRB, Hsu Y-H, Chasman DI, Johnson AD, Elks C, Albrecht E, Andrulis IL, Beesley J, Berenson GS, Bergmann S. DNA mismatch repair gene MSH6 implicated in determining age at natural menopause. Hum Mol Genet. 2013. doi:10.1093/hmg/ddt620.

9. Westra H-J, Peters MJ, Esko T, Yaghootkar H, Schurmann C, Kettunen J, Christiansen MW, Fairfax BP, Schramm K, Powell JE. Systematic identification of trans eQTLs as putative drivers of known disease associations. Nat Genet. 2013;45(10):1238-43

10. Schadt EE, Molony C, Chudin E, Hao K, Yang X, Lum PY, Kasarskis A, Zhang B, Wang S, Suver C. Mapping the genetic architecture of gene expression in human liver. PLoS Biol. 2008;6(5):e107.

11. Innocenti F, Cooper GM, Stanaway IB, Gamazon ER, Smith JD, Mirkov S, Ramirez J, Liu W, Lin YS, Moloney C. Identification, replication, and functional fine-mapping of expression quantitative trait loci in primary human liver tissue. PLoS Genet. 2011;7(5):e1002078. 
12. Lonsdale J, Thomas J, Salvatore M, Phillips R, Lo E, Shad S, Hasz R, Walters G, Garcia F, Young N. The Genotype-Tissue Expression (GTEx) project. Nat Genet. 2013;45(6):580-5.

13. Global Lipids Genetics Consortium. Discovery and refinement of loci associated with lipid levels. Nat Genet. 2013. doi:10.1038/ng.2797.

14. Pandit J, Danley DE, Schulte GK, Mazzalupo S, Pauly TA, Hayward CM, Hamanaka ES, Thompson JF, Harwood HJ. Crystal structure of human squalene synthase. A key enzyme in cholesterol biosynthesis. J Biol Chem. 2000;275(39):30610-7.

15. Chalasani N, Guo X, Loomba R, Goodarzi MO, Haritunians T, Kwon S, Cui J, Taylor KD, Wilson L, Cummings OW. Genome-wide association study identifies variants associated with histologic features of nonalcoholic fatty liver disease. Gastroenterology. 2010;139(5):1567-76. e1566.

16. Stein EA, Bays H, O'Brien D, Pedicano J, Piper E, Spezzi A. Lapaquistat acetate development of a squalene synthase inhibitor for the treatment of hypercholesterolemia. Circulation. 2011;123(18):1974-85.

17. Schönbeck U, Libby P. Inflammation, immunity, and HMG-CoA reductase inhibitors statins as antiinflammatory agents? Circulation. 2004;109(21 suppl 1): $\|-18-26$.

18. Dehghan A, Yang Q, Peters A, Basu S, Bis JC, Rudnicka AR, Kavousi M, Chen M-H, Baumert J, Lowe GDO. Association of novel genetic loci with circulating fibrinogen levels a genome-wide association study in 6 population-based cohorts. Circ Cardiovasc Genet. 2009;2(2):125-33.

19. Speliotes EK, Willer CJ, Berndt SI, Monda KL, Thorleifsson G, Jackson AU, Allen HL, Lindgren CM, Luan J, Mägi R. Association analyses of 249,796 individuals reveal 18 new loci associated with body mass index. Nat Genet. 2010;42(11):937-48.

20. Locke AE, Kahali B, Berndt SI, Justice AE, Pers TH, Day FR, Powell C, Vedantam S, Buchkovich ML, Yang J. Genetic studies of body mass index yield new insights for obesity biology. Nature. 2015;518(7538):197-206.

21. Timpson NJ, Nordestgaard BG, Harbord RM, Zacho J, Frayling TM, Tybjærg-Hansen A, Smith GD. C-reactive protein levels and body mass index: elucidating direction of causation through reciprocal Mendelian randomization. Int J Obes. 2011;35(2):300-8.

22. Ligthart S, de Vries PS, Uitterlinden AG, Hofman A, Franco OH, Chasman DI, Dehghan A, CHARGE Inflammation working group. Pleiotropy among common genetic loci identified for cardiometabolic disorders and C-Reactive protein. PLoS One. 2015;10(3):e0118859.

23. van Diepen JA, Berbée JFP, Havekes LM, Rensen PCN. Interactions between inflammation and lipid metabolism: relevance for efficacy of antiinflammatory drugs in the treatment of atherosclerosis. Atherosclerosis. 2013;228(2):306-15.

24. Hsu YH, Chen X. Identifying Pleiotropic Genetic Effects: A Two-Stage Approach Using Genome-Wide Association Meta-Analysis Data. https://sites. google.com/site/multivariateyihsianghsu/. Accessed 7 June 2016.

25. The Multi-Phenotype GWAS Analysis For Pleiotropic Genetic Effects [https:// sites.google.com/site/multivariateyihsianghsu/]. Accessed 7 June 2016.

26. Hofman A, Murad SD, van Duijn CM, Franco OH, Goedegebure A, Ikram MA Klaver CCW, Nijsten TEC, Peeters RP, Stricker BHC. The Rotterdam Study: 2014 objectives and design update. Eur J Epidemiol. 2013;28(11):889-926.

27. Almqvist C, Adami H-O, Franks PW, Groop L, Ingelsson E, Kere J, Lissner L, Litton J-E, Maeurer M, Michaëlsson K. LifeGene-a large prospective population-based study of global relevance. Eur J Epidemiol. 2011;26(1):67-77.

28. Voight BF, Kang HM, Ding J, Palmer CD, Sidore C, Chines PS, Burtt NP, Fuchsberger C, Li Y, Erdmann J. The metabochip, a custom genotyping array for genetic studies of metabolic, cardiovascular, and anthropometric traits. PLoS Genet. 2012;8(8):e1002793.

\section{Submit your next manuscript to BioMed Central and we will help you at every step:}

- We accept pre-submission inquiries

- Our selector tool helps you to find the most relevant journal

- We provide round the clock customer support

- Convenient online submission

- Thorough peer review

- Inclusion in PubMed and all major indexing services

- Maximum visibility for your research

Submit your manuscript at www.biomedcentral.com/submit
( ) BioMed Central 